\title{
A GENERALIZATION OF THE BANACH-STEINHAUS THEOREM FOR FINITE PART LIMITS
}

\author{
RICARDO ESTRADA AND JASSON VINDAS
}

\begin{abstract}
It is well known, as follows from the Banach-Steinhaus theorem, that if a sequence $\left\{y_{n}\right\}_{n=1}^{\infty}$ of linear continuous functionals in a Fréchet space converges pointwise to a linear functional $Y$, $Y(x)=\lim _{n \rightarrow \infty}\left\langle y_{n}, x\right\rangle$ for all $x$, then $Y$ is actually continuous. In this article we prove that in a Fréchet space the continuity of $Y$ still holds if $Y$ is the finite part of the limit of $\left\langle y_{n}, x\right\rangle$ as $n \rightarrow \infty$. We also show that the continuity of finite part limits holds for other classes of topological vector spaces, such as $L F$-spaces, $D F S$-spaces, and $D F S{ }^{*}$-spaces, and give examples where it does not hold.
\end{abstract}

\section{INTRODUCTION}

Let $X$ be a topological vector space over $K, K$ being $\mathbb{R}$ or $\mathbb{C}$. We denote as $X^{\prime}$ the dual space, that is, the space of continuous linear functionals on $X$; the evaluation of $y \in X^{\prime}$ on $x \in X$ will be denoted as $\langle y, x\rangle$ or as $y(x)$; we shall denote as $X_{\mathrm{al}}^{\prime}$ the algebraic dual of $X$, but if $z \in X_{\text {al }}^{\prime}$ we denote evalutions as $z(x)$ only.

Let $\left\{y_{n}\right\}_{n=1}^{\infty}$ be a sequence of elements of $X^{\prime}$ and suppose that

$$
\lim _{n \rightarrow \infty}\left\langle y_{n}, x\right\rangle=Y(x),
$$

exists for each $x \in X$, thus defining a function $Y: X \rightarrow K$. It is clear that $Y$ is linear, an element of $X_{\mathrm{al}}^{\prime}$, and simple examples show that $Y$ does not have to be continuous, that is, maybe $Y \notin X^{\prime}$. However, it is well known $[9,12]$ that if $X$ is barreled, in particular if $X$ is a Fréchet space or an $L F$ space, then one must have that $Y \in X^{\prime}$; this result is quite important in the theory of distributions since the usual spaces of test functions are barreled and thus (1.1) provides a method, rather frequently employed, to construct new distributions as limits.

2010 Mathematics Subject Classification. 46A04, 46A13.

Key words and phrases. Finite part limits, Hadamard finite part, BanachSteinhaus theorem.

R. Estrada gratefully acknowledges support from NSF, through grant number 0968448 . 
Our aim is to consider the continuity of $Y$ in case the standard ${ }^{1}$ finite part of the limit

$$
\text { F.p. } \lim _{n \rightarrow \infty}\left\langle y_{n}, x\right\rangle=Y(x)
$$

exists for each $x \in X$. We will show that $Y \in X^{\prime}$ in case $X$ is a Fréchet space or in case it is an inductive limit of Fréchet spaces. Naturally several distributions are defined as finite parts, so such a result would be very useful.

The meaning of (1.2) is that for each $x \in X$ there is $k=k_{x} \in \mathbb{N}_{0}=$ $\mathbb{N} \cup\{0\}$, exponents $\alpha_{1}>\cdots>\alpha_{k}>0$, scalars $R_{\alpha_{1}}(x), \ldots, R_{\alpha_{k}}(x) \in$ $K \backslash\{0\}$, and $z_{n}(x) \in K$ for $n \geq 0$ such that

$$
\left\langle y_{n}, x\right\rangle=n^{\alpha_{1}} R_{\alpha_{1}}(x)+\cdots+n^{\alpha_{k}} R_{\alpha_{k}}(x)+z_{n}(x),
$$

where

$$
\lim _{n \rightarrow \infty} z_{n}(x)=Y(x) .
$$

Observe that $k_{x}$ could be 0 , meaning that $\left\langle y_{n}, x\right\rangle=z_{n}(x)$ converges to $Y(x)$.

We call $n^{\alpha_{1}} R_{\alpha_{1}}(x)+\cdots+n^{\alpha_{k}} R_{\alpha_{k}}(x)$ the infinite part of $\left\langle y_{n}, x\right\rangle$ as $n \rightarrow \infty$ and $z_{n}(x)$ the finite part. Clearly the infinite and finite part, if they exist, are uniquely determined, so that the finite part of the limit, if it exists, is likewise uniquely determined.

It is important to point out that maybe $\sup \left\{k_{x}: x \in X\right\}=\infty$ and that the set of exponents,

$$
\bigcup_{x \in X}\left\{\alpha_{j}: 1 \leq j \leq k_{x}\right\}
$$

does not have to be finite. We shall show that when $X$ is a Fréchet space then $\sup \left\{k_{x}: x \in X\right\}<\infty$ and actually the set (1.5) is finite, but give examples in other types of spaces where these results do not hold.

We shall also show that the $R_{\alpha}$ admit extensions as elements of the algebraic dual $X_{\mathrm{al}}^{\prime}$, and show that while in general they are not continuous, they must belong to $X^{\prime}$ when $X$ is a Fréchet space or an inductive limit of Fréchet spaces.

The plan of the article is as follows. In Section 2 we give some basic facts about finite parts that hold in any topological vector space. The central part of the article is Section 3, where we study finite parts in a Fréchet space. Extensions to more general finite parts and to more general topological vector spaces are considered in Sections 4 and 5, respectively. Finally we present several illustrations in Section 6.

\footnotetext{
${ }^{1}$ We shall consider more complicated finite part limits later on.
} 


\section{General Results}

We shall first consider several results that hold in any topological vector space.

Thus let $\left\{y_{n}\right\}_{n=1}^{\infty}$ be a sequence of elements of the dual space $X^{\prime}$ of the topological vector space $X$, and suppose that for each $x \in X$ the finite part of the limit

$$
Y(x)=\text { F.p. } \lim _{n \rightarrow \infty}\left\langle y_{n}, x\right\rangle,
$$

exists, or, in other words, that the evaluation $\left\langle y_{n}, x\right\rangle$ can be decomposed as

$$
\left\langle y_{n}, x\right\rangle=u_{n}(x)+z_{n}(x),
$$

with the infinite part of the form

$$
u_{n}(x)=n^{\alpha_{1}} R_{\alpha_{1}}(x)+\cdots+n^{\alpha_{k}} R_{\alpha_{k}}(x),
$$

where $\alpha_{1}>\cdots>\alpha_{k}>0$, and $R_{\alpha_{1}}(x), \ldots, R_{\alpha_{k}}(x) \in K \backslash\{0\}$, and with the finite part, $z_{n}(x)$, such that the limit

$$
Y(x)=\lim _{n \rightarrow \infty} z_{n}(x)
$$

exists.

The following result is very easy to prove, but it is also very important.

Lemma 2.1. The decomposition (2.2) in finite and infinite parts is unique.

It is convenient to define $R_{\alpha}(x)$ for all $\alpha>0$ and all $x \in X$. We just put $R_{\alpha}(x)=0$ if $\alpha$ is not one of the exponents $\alpha_{1}, \ldots, \alpha_{k}$ in the expression of the infinite part of $\left\langle y_{n}, x\right\rangle$. This allows us to rewrite (2.3) as

$$
u_{n}(x)=\sum_{\alpha>0} n^{\alpha} R_{\alpha}(x)
$$

since only a finite number of terms of the uncountable sum do not vanish.

Lemma 2.2. If $Y(x)=$ F.p. $\lim _{n \rightarrow \infty}\left\langle y_{n}, x\right\rangle$ exists for all $x \in X$ then $Y$ is linear: $Y \in X_{\mathrm{al}}^{\prime}$.

Proof. Indeed, if $x_{1}, x_{2} \in X$ and $c \in K$, then $\left\langle y_{n}, x_{1}+c x_{2}\right\rangle$ admits the decomposition

$$
\begin{aligned}
\left\langle y_{n}, x_{1}+c x_{2}\right\rangle & =\left\langle y_{n}, x_{1}\right\rangle+c\left\langle y_{n}, x_{2}\right\rangle \\
& =\left(u_{n}\left(x_{1}\right)+c u_{n}\left(x_{2}\right)\right)+\left(z_{n}\left(x_{1}\right)+c z_{n}\left(x_{2}\right)\right) .
\end{aligned}
$$


Since

$$
u_{n}\left(x_{1}\right)+c u_{n}\left(x_{2}\right)=\sum_{\alpha>0} n^{\alpha}\left(R_{\alpha}\left(x_{1}\right)+c R_{\alpha}\left(x_{2}\right)\right),
$$

has the form of an infinite part, the Lemma 2.1 yields

$u_{n}\left(x_{1}+c x_{2}\right)=u_{n}\left(x_{1}\right)+c u_{n}\left(x_{2}\right), \quad z_{n}\left(x_{1}+c x_{2}\right)=z_{n}\left(x_{1}\right)+c z_{n}\left(x_{2}\right)$, and consequently $Y\left(x_{1}+c x_{2}\right)$ equals

$$
\lim _{n \rightarrow \infty} z_{n}\left(x_{1}+c x_{2}\right)=\lim _{n \rightarrow \infty}\left(z_{n}\left(x_{1}\right)+c z_{n}\left(x_{2}\right)\right)
$$

that is, $Y\left(x_{1}\right)+c Y\left(x_{2}\right)$.

If we now use the fact that $R_{\alpha}(x)=$ F.p. $\lim _{n \rightarrow \infty} n^{-\alpha}\left\langle y_{n}, x\right\rangle$, or employ (2.6), we immediately obtain the ensuing result.

Lemma 2.3. For any $\alpha>0$ the function $R_{\alpha}$ is linear, $R_{\alpha} \in X_{\mathrm{al}}^{\prime}$.

In general $Y$ nor all $R_{\alpha}$ will not be continuous, as the Example 6.5 shows.

\section{Finite parts in a FrÉchet space}

We shall now consider the continuity and structure of finite parts in a Fréchet space. We start with some useful preliminary results.

Lemma 3.1. Let $X$ be a Fréchet space and let $\left\{y_{n}\right\}_{n=0}^{\infty}$ be a sequence of non zero elements of $X^{\prime}$. Then there exists $x \in X$ such that $\left\langle y_{n}, x\right\rangle \neq 0$ $\forall n \in \mathbb{N}_{0}$.

Proof. Indeed, since $y_{n} \neq 0$ the kernel of $y_{n}, F_{n}=\left\{x \in X:\left\langle y_{n}, x\right\rangle=0\right\}$ is a closed proper subspace of $X$ and thus of first category. Hence $\cup_{n=0}^{\infty} F_{n} \neq X$.

Observe that this result fails in spaces that are not Fréchet. Consider, for example, the sequence $\{\delta(t-n)\}_{n=0}^{\infty}$ in the space $\mathcal{D}^{\prime}(\mathbb{R})$.

Recall that a function $f: W \rightarrow V$, where $W$ and $V$ are topological spaces, is called a Baire function of the first class if there exists a sequence of continuous functions from $W$ to $V,\left\{f_{n}\right\}_{n=0}^{\infty}$, such that $f(w)=\lim _{n \rightarrow \infty} f_{n}(w)$ for all elements $w \in W$.

Lemma 3.2. Let $X$ be a Fréchet space and let $A: X \rightarrow \mathbb{R}$ be a function that satisfies the following three properties:

1. A is a Baire function of the first class;

2. $A(x-y) \leq \max \{A(x), A(y)\}$;

3. $A(c x)=A(x)$ if $c \neq 0$.

Then $A$ is bounded above in $X$ and it actually attains its maximum. 
Proof. We shall first show that $A$ is bounded above. If $F$ is a subset of $X$, denote by $M_{F}=\sup \{A(x): x \in F\}$. If $U$ is a neighborhood of 0 , then 3 yields that $M_{X}=M_{U}$. Let now $V$ be any set with non empty interior; then $V-V$ is a neighborhood of 0 and thus 2 yields that $M_{X}=M_{V-V} \leq M_{V} \leq M_{X}$ so that $M_{X}=M_{V}$.

Let $\alpha_{n}$ be a sequence of continuous functions from $X$ to $\mathbb{R}$ that converges to $A$ everywhere. Then $X=\bigcup_{k=0}^{\infty}\left\{x \in X: \alpha_{n}(x) \leq k, \forall n\right\}$ so that there exists $k \in \mathbb{N}$ such that the set $V_{k}=\left\{x \in X: \alpha_{n}(x) \leq k \forall n\right\}$ has non empty interior. This yields that $M_{X}=M_{V_{k}} \leq k$, so that $A$ is bounded above by $k$ in the whole space $X$.

We should now show that there exists $\widetilde{x} \in X$ such that $A(\widetilde{x})=M_{X}$. If not, the function $B(x)=1 /\left(M_{X}-A(x)\right)$ satisfies the same three conditions as $A$, and from what we have already proved, $B$ must be bounded above by some constant $\lambda>0$; but this means that $A(x) \leq M_{X}-1 / \lambda$, for all $x \in X$, and consequently $M_{X} \leq M_{X}-1 / \lambda$, a contradiction.

We now apply the Lemma 3.2 to the study of finite parts. Indeed, let $\left\{y_{n}\right\}_{n=1}^{\infty}$ be a sequence of elements of the dual space $X^{\prime}$ of the Fréchet space $X$, and suppose that for each $x \in X$ the finite part of the limit $Y(x)=$ F.p. $\lim _{n \rightarrow \infty}\left\langle y_{n}, x\right\rangle$ exists. If the infinite part of $\left\langle y_{n}, x\right\rangle$ has the expression as a finite sum,

$$
u_{n}(x)=n^{\alpha_{1}} R_{\alpha_{1}}(x)+\cdots+n^{\alpha_{k}} R_{\alpha_{k}}(x)=\sum_{\alpha>0} n^{\alpha} R_{\alpha}(x),
$$

where $\alpha_{1}>\cdots>\alpha_{k}>0$, and $R_{\alpha_{1}}(x), \ldots, R_{\alpha_{k}}(x) \in K \backslash\{0\}$, define $A(x)=0$ if $u_{n}(x)=0$ and as

$$
A(x)=\alpha_{1}=\max \left\{\alpha>0: R_{\alpha}(x) \neq 0\right\},
$$

otherwise.

Lemma 3.3. The function $A$ is bounded above and attains its maximum in $X$.

Proof. It is enough to prove that $A$ satisfies the three conditions of the Lemma 3.2. However, condition 1 follows from the limit formula

$$
A(x)=\lim _{n \rightarrow \infty} \frac{\ln \left[\left|\left\langle y_{n}, x\right\rangle\right|+1\right]}{\ln n},
$$

while 2 and 3 are obvious.

The Lemma 3.3 not only means that if $\widetilde{\alpha}=\max \{A(x): x \in X\}$ then $R_{\alpha}(x)=0$ if $\alpha>\widetilde{\alpha}$, but it also means that $R_{\widetilde{\alpha}} \neq 0$. The linear 
form $R_{\widetilde{\alpha}}$ is actually continuous as follows from the Banach-Steinhaus theorem since

$$
R_{\widetilde{\alpha}}(x)=\lim _{n \rightarrow \infty} n^{-\widetilde{\alpha}_{1}}\left\langle y_{n}, x\right\rangle
$$

for each $x \in X$.

We can then replace $y_{n}$ by $y_{n}-n^{\widetilde{\alpha}} R_{\widetilde{\alpha}}$ and apply the same ideas as above. Therefore, for some integers $k$ we obtain exponents $\widetilde{\alpha}_{1}=\widetilde{\alpha}>$ $\cdots>\widetilde{\alpha}_{k}>0$ such that $R_{\alpha}(x)=0$ if $\alpha>\widetilde{\alpha}_{k}, \alpha \neq \widetilde{\alpha}_{j}, 1 \leq j<k$, while $R_{\widetilde{\alpha}_{j}}$ is continuous and $R_{\widetilde{\alpha}_{j}} \neq 0$ for $1 \leq j \leq k$. In principle one could think that this is possible for each $k \geq 0$, but if it were then we would obtain an infinite sequence of non zero continuous functionals $\left\{R_{\widetilde{\alpha}_{j}}\right\}_{j=0}^{\infty}$ and the Lemma 3.1 would give us the existence of $x^{*} \in X$ such that $R_{\widetilde{\alpha}_{j}}\left(x^{*}\right) \neq 0$ for all $j$, a contradiction, since for any $x \in X$ the set $\left\{\alpha>0: R_{\alpha}(x) \neq 0\right\}$ is finite. Summarizing, we have the following result.

Theorem 3.4. Let $\left\{y_{n}\right\}_{n=1}^{\infty}$ be a sequence of elements of the dual space $X^{\prime}$ of the Fréchet space $X$, and suppose that for each $x \in X$ the finite part of the limit $Y(x)=$ F.p. $\lim _{n \rightarrow \infty}\left\langle y_{n}, x\right\rangle$ exists. Then there exists $k \in \mathbb{N}$, exponents $\widetilde{\alpha}_{1}>\cdots>\widetilde{\alpha}_{k}>0$, and continuous non zero linear functionals $\left\{R_{\widetilde{\alpha}_{j}}\right\}_{j=1}^{k}$ such that for all $x \in X$

$$
\left\langle y_{n}, x\right\rangle=n^{\widetilde{\alpha}_{1}} R_{\widetilde{\alpha}_{1}}(x)+\cdots+n^{\widetilde{\alpha}_{k}} R_{\widetilde{\alpha}_{k}}(x)+z_{n}(x),
$$

where the finite part $z_{n}$ is continuous for all $n$ and where

$$
Y(x)=\lim _{n \rightarrow \infty} z_{n}(x),
$$

is also a continuous linear functional on $X$.

Proof. The only thing left to prove is the continuity of the $z_{n}$ 's and the continuity of $Y$. But the continuity of the $R_{\widetilde{\alpha}_{j}}$ 's yields the continuity of the $z_{n}$ 's because of (3.5) while the continuity of $Y$ follows from (3.6) and the Banach-Steinhaus theorem.

\section{More General Finite PARTS}

One can consider a general finite part limit process as follows. Let $\Lambda \cup\left\{\lambda_{0}\right\}$ be a topological space where $\lambda_{0} \in \bar{\Lambda} \backslash \Lambda$, and let $(\mathfrak{E}, \prec)$ be a totally ordered set. Let $B=\left\{\rho_{\alpha}\right\}_{\alpha \in \mathfrak{E}}$ be the "basic infinite functions," that is, a family of functions with the following properties:

(1) For each $\alpha \in \mathfrak{E}, \rho_{\alpha}: \Lambda \rightarrow(0, \infty)$, and $\lim _{\lambda \rightarrow \lambda_{0}} \rho_{\alpha}(\lambda)=\infty$;

(2) If $\alpha \prec \beta$ then $\rho_{\alpha}(\lambda)=o\left(\rho_{\beta}(\lambda)\right)$ as $\lambda \rightarrow \lambda_{0}$. 
Let $y_{\lambda} \in K$, where $K$ is $\mathbb{R}$ or $\mathbb{C}$, for each $\lambda \in \Lambda$. If we can write

$$
y_{\lambda}=u_{\lambda}+z_{\lambda}
$$

where the "infinite part" has the form

$$
u_{\lambda}=\sum_{j=1}^{k} R_{\alpha_{j}} \rho_{\alpha_{j}}(\lambda),
$$

where $\alpha_{1} \succ \cdots \succ \alpha_{k}$, and $R_{\alpha_{1}}, \ldots, R_{\alpha_{k}} \in K \backslash\{0\}$, and where the "finite part," $z_{\lambda}$, satisfies that the limit

$$
Y=\lim _{\lambda \rightarrow \lambda_{0}} z_{\lambda}
$$

exists, then we say that the finite part of the limit of $y_{\lambda}$ as $\lambda \rightarrow \lambda_{0}$ with respect to $\mathrm{B}$ exists and equals $Y$, and write ${ }^{2}$

$$
Y=\mathrm{F} \cdot \mathrm{p} \cdot \mathrm{B} \lim _{\lambda \rightarrow \lambda_{0}} y_{\lambda} \text {. }
$$

We have considered the standard system $B=\left\{\rho_{\alpha}\right\}_{\alpha>0}$ where $\Lambda=\mathbb{N}$, $\lambda_{0}=\infty$, and $\rho_{\alpha}(\lambda)=\lambda^{\alpha}$. Naturally one can consider the same standard system for functions defined in any unbounded set $\Lambda \subset(0, \infty)$, in particular for $\Lambda=(0, \infty)$.

We can also consider Hadamard finite part limits ${ }^{3}$, where the infinite basic functions are products of powers and powers of logarithms. Explicitly, let $\mathfrak{E}=[0, \infty)^{2} \backslash\{(0,0)\}$, with the order given by $\left(\alpha_{1}, \beta_{1}\right) \prec$ $\left(\alpha_{2}, \beta_{2}\right)$ if $\alpha_{1}<\alpha_{2}$ or if $\alpha_{1}=\alpha_{2}$ and $\beta_{1}<\beta_{2}$. Here $\Lambda \subset(1, \infty)$, and the basic infinite functions $\mathrm{B}=\left\{\rho_{(\alpha, \beta)}\right\}_{(\alpha, \beta) \in \mathfrak{E}}$ are given as $\rho_{(\alpha, \beta)}(\lambda)=$ $\lambda^{\alpha} \ln ^{\beta} \lambda$.

We can also take a set $\epsilon \subset(0,1)$ and consider limits as $\varepsilon \in \epsilon$ tends to 0 . For standard finite part limits $\mathfrak{E}=(0, \infty)$ and the basic infinite functions are $\rho_{\alpha}(\varepsilon)=\varepsilon^{-\alpha}$; for Hadamard finite part limits, $\mathfrak{E}=[0, \infty)^{2} \backslash\{(0,0)\}$ and $\rho_{(\alpha, \beta)}(\varepsilon)=\varepsilon^{-\alpha}|\ln \varepsilon|^{\beta}$.

The continuity of the finite part of the limit in Fréchet spaces, Theorem 3.4, will also hold for these more general systems of basic infinite functions. This is of course the case for standard finite limits. For Hadamard finite parts the proof can be modified as follows. Indeed, let $X$ be a Fréchet space, $\Lambda \subset(1, \infty)$ is an unbounded set and $y_{\lambda} \in X^{\prime}$

\footnotetext{
${ }^{2}$ Let $V$ be the vector space of all functions of the form $\sum_{j=1}^{k} c_{j} \rho_{\alpha_{j}}+\mu$ with $\lim _{\lambda \rightarrow \lambda_{0}} \mu(\lambda)=0$. The triple $N=(\Lambda, K, V)$ forms a neutrix in the sense of van der Corput [13]. In his terminology, the finite part limit (4.4) coincides with the neutrix value $y_{N}$.

${ }^{3}$ Hadamard was probably the first to use finite parts; in his 1923 work [7], he employs them to find fundamental solutions of partial differential equations.
} 
for each $\lambda \in \Lambda$. Suppose that for each $x \in X$ the evaluation $\left\langle y_{\lambda}, x\right\rangle$ can be written as

$$
\left\langle y_{\lambda}, x\right\rangle=u_{\lambda}(x)+z_{\lambda}(x),
$$

where the infinite part has the ensuing form for some $k=k_{x}$,

$$
u_{\lambda}(x)=\lambda^{\alpha_{1}} \ln ^{\beta_{1}} \lambda R_{\left(\alpha_{1}, \beta_{1}\right)}(x)+\cdots+\lambda^{\alpha_{k}} \ln ^{\beta_{k}} \lambda R_{\left(\alpha_{k}, \beta_{k}\right)}(x),
$$

where the exponents $\left(\alpha_{j}, \beta_{j}\right) \in \mathfrak{E}$ satisfy $\left(\alpha_{1}, \beta_{1}\right) \succ \cdots \succ\left(\alpha_{k}, \beta_{k}\right)$, where $R_{\left(\alpha_{1}, \beta_{1}\right)}(x), \ldots, R_{\left(\alpha_{k}, \beta_{k}\right)}(x) \in K \backslash\{0\}$, and where the finite part, $z_{\lambda}(x)$, satisfies that the limit

$$
Y(x)=\lim _{\lambda \rightarrow \infty, \lambda \in \Lambda} z_{\lambda}(x),
$$

exists. As before, we set $R_{(\alpha, \beta)}(x)=0$ if $(\alpha, \beta) \neq\left(\alpha_{j}, \beta_{j}\right)$ for $1 \leq j \leq$ $k_{x}$. Then we have the following generalization of the Lemma 3.3.

Lemma 4.1. Let

$$
E(x)=(A(x), B(x))=\max \left\{(\alpha, \beta) \in \mathfrak{E}: R_{(\alpha, \beta)}(x) \neq 0\right\} .
$$

Then $E$ attains its maximum, $\left(\alpha^{*}, \beta^{*}\right)$, and $R_{\left(\alpha^{*}, \beta^{*}\right)}$ is continuous and not zero.

Proof. The proof of the Lemma 3.3 applies to $A$, so there exists $\alpha^{*}=$ $\max _{x \in X} A(x)$. For this exponent $\alpha^{*}$ we consider the function given by $B^{*}(x)=0$ if $R_{\left(\alpha^{*}, \beta\right)}(x)=0$ for all $\beta$ and otherwise by

$$
B^{*}(x)=\max \left\{\beta: R_{\left(\alpha^{*}, \beta\right)}(x) \neq 0\right\} .
$$

The Lemma 3.2 yields the existence of $\beta^{*}=\max _{x \in X} B^{*}(x)$ because

$$
B^{*}(x)=\lim _{\lambda \rightarrow \infty} \frac{\ln \left[\lambda^{-\alpha^{*}}\left|\left\langle y_{\lambda}, x\right\rangle\right|+1\right]}{\ln \ln \lambda} .
$$

Since

$$
R_{\left(\alpha^{*}, \beta^{*}\right)}(x)=\lim _{\lambda \rightarrow \infty}\left(\lambda^{-\alpha^{*}} \ln ^{-\beta^{*}} \lambda\right)\left\langle y_{\lambda}, x\right\rangle,
$$

the continuity of $R_{\left(\alpha^{*}, \beta^{*}\right)}$ follows.

Therefore we obtain that the Theorem 3.4 holds for Hadamard finite parts.

These ideas can be further generalized. Let us take $m$ positive functions $F_{1}, F_{2}, F_{3}, \ldots, F_{m}$ defined on an unbounded set $\Lambda \subset(1, \infty)$, each of them tending to $\infty$ as $\lambda \rightarrow \infty$ and such that $F_{j+1}(\lambda)=o\left(F_{j}^{\alpha}(\lambda)\right)$ as $\lambda \rightarrow \infty$ for all $\alpha>0$. We now consider $\mathfrak{E}=[0, \infty)^{m} \backslash\{(0,0, \ldots, 0)\}$ with the lexicographical order $\prec$. Set $\mathbf{F}=\left(F_{1}, F_{2}, \ldots, F_{m}\right)$ and if $\vec{\alpha} \in \mathfrak{E}$ write $\mathbf{F}^{\vec{\alpha}}=F_{1}^{\alpha_{1}} F_{2}^{\alpha_{2}} \cdots F_{m}^{\alpha_{m}}$, where $\vec{\alpha}=\left(\alpha_{1}, \alpha_{2}, \ldots, \alpha_{m}\right)$. We now choose the basic infinite functions as $\mathrm{B}=\left\{\mathbf{F}^{\vec{\alpha}}\right\}_{\vec{\alpha} \in \mathfrak{E}}$. If $y_{\lambda} \in X^{\prime}$ 
for each $\lambda \in \Lambda$, where $X$ is again a Fréchet space, and for each $x \in X$ the evaluation $\left\langle y_{\lambda}, x\right\rangle$ can be decomposed as in (4.5) where the infinite part is now taken of the form (for some $k=k_{x}$,)

$$
u_{\lambda}(x)=\mathbf{F}^{\vec{\alpha}_{1}}(\lambda) R_{\vec{\alpha}_{1}}(x)+\cdots+\mathbf{F}^{\vec{\alpha}_{k}}(\lambda) R_{\vec{\alpha}_{1}}(x),
$$

with $\vec{\alpha}_{1} \succ \cdots \succ \vec{\alpha}_{k}$, and $R_{\vec{\alpha}_{1}}(x), \ldots, R_{\vec{\alpha}_{k}}(x) \in K \backslash\{0\}$, and where $z_{\lambda}(x)$ satisfies (4.7), we can then define the finite part limit of $y_{\lambda}(x)$ as $Y(x)$. Defining $R_{\vec{\alpha}}(x)=0$ if $\vec{\alpha}$ does not occur in (4.12), the proof of Lemma 4.1 can be readily adapted to show that the function $\mathbf{A}(x)=$ $\max \left\{\vec{\alpha} \in \mathfrak{E}: R_{\vec{\alpha}}(x) \neq 0\right\}$ also attains its maximum, $\vec{\alpha}^{*}$, and that $R_{\vec{\alpha}^{*}} \in$ $X^{\prime} \backslash\{0\}$. This leads to a general version of Theorem 3.4 for finite part

limits with respect to the system of infinite functions $B=\left\{\mathbf{F}^{\vec{\alpha}}\right\}_{\vec{\alpha} \in \mathfrak{E}}$. Naturally, the Hadamard finite part corresponds to the choices $m=2$, $F_{1}(\lambda)=\lambda$, and $F_{2}(\lambda)=\log \lambda$.

\section{Other types of TOPOLOGICAL VECTOR SPACES}

The continuity of finite part limits holds not only in Fréchet spaces, but in other types of spaces, those that carry a final locally convex topology given by a family of Fréchet spaces [9]. Indeed, let $\left(X_{i}, u_{i}\right)_{I}$ be a family of Fréchet spaces and linear mappings $u_{i}: X_{i} \rightarrow X$ for each $i \in I$. If $X$ is provided with the finest locally convex topology that makes all mappings $u_{i}$ continuous, the continuity of the finite part limits and the $R_{\vec{\alpha}}$ 's follows at once from the fact that $y \in X^{\prime}$ if and only if $y \circ u_{i} \in X_{i}^{\prime}, \forall i \in I$. In particular, the result holds for any inductive limit of an inductive system of Fréchet spaces. Important instances of such inductive limits are those that can be written as countable inductive unions of Fréchet spaces, such as the $L F$-spaces, the DFS-spaces, and the $D F S^{*}$-spaces [11].

In these more general spaces, however, the set of exponents for which $R_{\vec{\alpha}}(x) \neq 0$ does not have to be finite, not bounded, in general (see Examples 6.2, 6.3, and 6.4 below).

Theorem 5.1. Let $X$ be a locally convex space that is the inductive limit of a system of Fréchet spaces. Let $\Lambda$ be an unbounded subset of $(1, \infty)$ and for each $\lambda \in \Lambda$ let $y_{\lambda} \in X^{\prime}$. Suppose that for each $x \in X$ the finite part of the limit $Y(x)=$ F.p. $\lim _{\lambda \rightarrow \infty}\left\langle y_{\lambda}, x\right\rangle$ exists. Then $Y \in X^{\prime}$.

Likewise, $R_{\vec{\alpha}}$ is continuous for each $\vec{\alpha}$, but while for each $x \in X$ the set $\left\{\vec{\alpha}: R_{\vec{\alpha}}(x) \neq 0\right\}$ is finite, the set $\left\{\vec{\alpha}: R_{\vec{\alpha}} \neq 0\right\}$ could be infinite and not bounded above. 


\section{EXAMPLES}

In order to better understand our results, it is useful to look at several examples.

Example 6.1. The best known example of finite parts are the distributions constructed as the finite part of divergent integrals $[4,10]$. Suppose $G$ is a homogenous continuous function in $\mathbb{R}^{d} \backslash\{\mathbf{0}\}$, homogenous of degree $\lambda \in \mathbb{R}$. Then $G$ gives a well defined distribution of the space $\mathcal{D}^{\prime}\left(\mathbb{R}^{d} \backslash\{\mathbf{0}\}\right)$, which without loss of generality we can still denote by $G$, as $\langle G, \phi\rangle=\int_{\mathbb{R}^{d} \backslash\{\mathbf{0}\}} G(\mathbf{x}) \phi(\mathbf{x}) \mathrm{d} \mathbf{x}$, for $\phi \in \mathcal{D}\left(\mathbb{R}^{d} \backslash\{\mathbf{0}\}\right)$. When $\lambda<-d$ then the integral $\int_{\mathbb{R}^{d}} G(\mathbf{x}) \phi(\mathbf{x}) \mathrm{d} \mathbf{x}$, would be divergent in general if $\phi \in \mathcal{D}\left(\mathbb{R}^{d}\right)$ and thus there is no canonical distribution corresponding to $G$ in $\mathcal{D}^{\prime}\left(\mathbb{R}^{d}\right)$. One can, however, define the distribution F.p. $(G)$, the radial finite part ${ }^{4}$ of $G$ by setting for $\phi \in \mathcal{D}\left(\mathbb{R}^{d}\right)$

$$
\langle\text { F.p. }(G), \phi\rangle=\text { F.p. } \lim _{n \rightarrow \infty} \int_{|\mathbf{x}| \geq 1 / n} G(\mathbf{x}) \phi(\mathbf{x}) \mathrm{d} \mathbf{x},
$$

a standard finite part if $-\lambda \notin \mathbb{N}$, and a Hadamard finite part if $\lambda$ is an integer. Similar ideas are needed to construct thick distributions from locally integrable functions [15] .

Example 6.2. Let $\mathbb{D}=\{z \in \mathbb{C}:|z|<1\}$ be the unit disc in $\mathbb{C}$. Let $H_{k}$ be the Banach space of functions continuous in $\overline{\mathbb{D}} \backslash\{0\}$, analytic in $\mathbb{D} \backslash\{0\}$, and that have a pole at $z=0$ of order $k$; the norm being $\|f\|=\max _{|z| \leq 1}|z|^{k}|f(z)|$. Let $H$ be the inductive limit of the $H_{k}$ as $k \rightarrow \infty$. Consider the functionals $y_{n} \in H^{\prime}$ given as

$$
\left\langle y_{n}, f\right\rangle=f(1 / n)
$$

that is, $y_{n}=\delta(z-1 / n)$. For each $f \in H$ the finite part of the limit F.p. $\lim _{n \rightarrow \infty}\left\langle y_{n}, f\right\rangle=Y(f)$ exists, and equals the finite part of $f$ at $z=0$; in fact, if $f(z)=\sum_{j=1}^{k} a_{j} z^{-j}+g(z)$, where $g$ is analytic at 0 , then

$$
\left\langle y_{n}, f\right\rangle=\sum_{j=1}^{k} a_{j} n^{j}+g(1 / n) .
$$

Observe that the infinite part is $\sum_{j=1}^{k} a_{j} n^{j}$, which has arbitrary large exponents; here the set (1.5) is infinite. Also $Y(f)=g(0)$, the usual

\footnotetext{
${ }^{4}$ If instead of removing balls of small radius, solids of other shapes are removed one obtains a different finite part distribution [5, 8, 14], an important fact in the numerical solution of integral equations [5]. The known formulas for the distributional derivatives of inverse power fields $[6]$ and the corresponding finite parts $[2,3]$ hold for radial finite parts.
} 
finite part of the analytic function at the pole. Our results will yield the continuity of $Y$, but one can prove this directly, for example, by observing that $Y(f)=(2 \pi i)^{-1} \oint_{|z|=r} z^{-1} f(z) \mathrm{d} z$ for any $r \in(0,1]$.

Interestingly, if $X$ is the space of all analytic function in $\mathbb{D} \backslash\{0\}$ with its standard topology, then $H$ is dense in $X$ and the $y_{n}$ 's and $Y$ admit continuous extensions to $X^{\prime}$, but the extension of $Y$ is not the finite part of the limit of the extensions of the $y_{n}$ 's.

Example 6.3. Let us consider the distributions

$$
f_{n}(x)=\sum_{k=1}^{\infty}\left(n^{1 / k}+1\right)^{k^{2}} \delta(x-k)
$$

of the space $\mathcal{D}^{\prime}(\mathbb{R})$. If $\phi \in \mathcal{D}(\mathbb{R})$ satisfies $\operatorname{supp} \phi \subset(-\infty, k+1)$ then the infinite part of $\left\langle f_{n}, \phi\right\rangle$ is the sum of $k^{2}-1$ terms, corresponding to the exponents $\alpha_{j}=j / k$ for $1 \leq j \leq k^{2}$. Hence the finite part of the limit is the Dirac comb

$$
\text { F.p. } \lim _{n \rightarrow \infty} f_{n}(x)=\sum_{k=1}^{\infty} \delta(x-k),
$$

while $R_{\alpha}(x) \neq 0$ precisely when $\alpha$ is a positive rational number; and actually if $k$ is the smallest integer for which $\alpha=j / k$ and $j \leq k^{2}$, then

$$
R_{j / k}(x)=\sum_{q=1}^{\infty}\left(\begin{array}{c}
q^{2} k^{2} \\
q j
\end{array}\right) \delta(x-q k) .
$$

One could represent the infinite part of $f_{n}(x)$ as the infinite sum $\sum_{\alpha \in \mathbb{Q}_{+}} R_{\alpha}(x)$; the set of exponents $\alpha$ for which $R_{\alpha} \neq 0$ is the infinite unbounded set $\mathbb{Q}_{+}$, but upon evaluation on a test function the sum becomes finite since $\left\langle R_{\alpha}, \phi\right\rangle \neq 0$ for only a finite set of exponents.

In the previous example $X=\mathcal{D}(\mathbb{R})$ is an $L F$ space, and it is not hard to see that in an $L F$ space the set of exponents $\alpha$ for which $R_{\alpha} \neq 0$ is countable at the most. We can easily construct an example where this set of exponents is the whole $(0, \infty)$.

Example 6.4. Let $X$ be the space of functions $f:(0, \infty) \rightarrow \mathbb{R}$ such that the set $\{\alpha \in(0, \infty): f(\alpha) \neq 0\}$ is finite. We give $X$ the inductive limit topology of the system $\left(\mathbb{R}^{F}, i_{F}\right)$, where $F$ is a finite subset of $(0, \infty), F \nearrow$, and if $f \in \mathbb{R}^{F}$, then $i_{F}(f)=f_{F} \in X$ is given by $f_{F}(\alpha)=f(\alpha)$ if $\alpha \in F$ and $f_{F}(\alpha)=0$ if $\alpha \notin F$. Theorem 5.1 applies in $X$. 
Let $y_{n} \in X^{\prime}$ be given as

$$
y_{n}(x)=\sum_{\alpha \in(0, \infty)} n^{\alpha} \delta(x-\alpha)
$$

that is $\left\langle y_{n}, f\right\rangle$ is the finite sum $\sum_{\alpha \in(0, \infty)} n^{\alpha} f(\alpha)$. Then $R_{\alpha}(x)=$ $\delta(x-\alpha)$ for all $\alpha \in(0, \infty)$, so that the set $(1.5)$ is the whole $(0, \infty)$. Notice also that F.p. $\lim _{n \rightarrow \infty} y_{n}(x)=0$.

Example 6.5. Consider the space $X$ whose elements are the continuous functions in $[0,1]$, with the topology of pointwise convergence on $[0,1]$. If $0<\beta<1$, let us consider the functional $f_{n} \in X^{\prime}$ given by

$$
f_{n}(x)=\sum_{k=0}^{n-1} \delta\left(x-\frac{k+\beta}{n}\right)
$$

that is,

$$
\left\langle f_{n}, \phi\right\rangle=\sum_{k=0}^{n-1} \phi\left(\frac{k+\beta}{n}\right),
$$

for $\phi \in X$. Then the Euler-Maclaurin formula [1] yields

$$
\left\langle f_{n}, \phi\right\rangle=n \int_{0}^{1} \phi(x) \mathrm{d} x+B_{1}(\beta)(\phi(1)-\phi(0)),
$$

where $B_{1}(x)=x-1 / 2$ is the Bernoulli polynomial of order 1 . The finite part is

$$
\text { F.p. } \lim _{n \rightarrow \infty} f_{n}(x)=B_{1}(\beta)(\delta(x-1)-\delta(x)),
$$

which is actually continuous in $X$, but $R_{1}$, the coefficient of $n$ in the infinite part of $\left\langle f_{n}, \phi\right\rangle$ is not continuous since it is given by $\phi \rightsquigarrow$ $\int_{0}^{1} \phi(x) \mathrm{d} x$, which belongs to $X_{\text {al }}^{\prime}$ but not to $X^{\prime}$.

Example 6.6. Let $\Omega$ be a complex region and let $\xi \in \Omega$. Suppose $y_{\omega} \in X^{\prime}$ is weakly-* analytic in $\omega \in \Omega \backslash\{\xi\}$, that is, for each $x \in X$ the function $\left\langle y_{\omega}, x\right\rangle$ is analytic in $\Omega \backslash\{\xi\}$. Suppose also that $\left\langle y_{\omega}, x\right\rangle$ has a pole at $\omega=\xi$ for each $x$.

If $X$ is a Fréchet space then there exists a fixed number $N$ such that the order of the pole is $N$ at the most for all $x$, and the finite part

$$
y_{\xi}^{*}(x)=\text { F.p. } \lim _{n \rightarrow \infty}\left\langle y_{\xi+1 / n}, x\right\rangle,
$$

is an element of $X^{\prime}$.

If $X$ is an inductive limit of Fréchet spaces, then $y_{\xi}^{*}$ is still continuous, but the order of the pole of $\left\langle y_{\omega}, x\right\rangle$ at $\xi$ does not have to be bounded, 
that is, maybe $R_{k}(x)=$ F.p. $\lim _{n \rightarrow \infty} n^{-k}\left\langle y_{\xi+1 / n}, x\right\rangle$ does not vanish in $X$ for an infinite number of values of $k$.

\section{REFERENCES}

[1] Estrada, R., On the Euler-Maclaurin asymptotic formula, Bol. Soc. Mat. Mex. 3 (1997), 117-133.

[2] Estrada, R. and Kanwal, R. P., Regularization and distributional derivatives of $\left(x_{1}^{2}+\cdots x_{p}^{2}\right)^{n / 2}$ in $\mathcal{D}^{\prime}\left(\mathbb{R}^{p}\right)$, Proc. Roy. Soc. London A 401 (1985), 281-297.

[3] Estrada, R. and Kanwal, R. P., Regularization, pseudofunction, and Hadamard finite part, J. Math. Anal. Appl. 141 (1989), 195-207.

[4] Estrada, R. and Kanwal, R.P., A distributional approach to asymptotics. Theory and applications, Second Edition, Birkhäuser, Boston, 2002.

[5] Farassat, F., Introduction to generalized functions with applications in aerodynamics and aeroacoustics, NASA Technical Paper 3248 (Hampton, VA: NASA Langley Research Center) (1996); http://ntrs.nasa.gov.

[6] Frahm, C.P., Some novel delta-function identities, Am. J. Phys. 51 (1983), $826-829$.

[7] Hadamard, J., Lectures on Cauchy's Problem in Linear Partial Differential Equations, Yale University Press, New Haven, 1923, (reprinted by Dover, New York, 1953).

[8] Hnizdo, V., Generalized second-order partial derivatives of $1 / r$, Eur. J. Phys. 32 (2011), 287-297.

[9] Horváth, J., Topological Vector Spaces and Distributions, vol. I., AddisonWesley, Reading, Massachusetts, 1966.

[10] Kanwal, R.P., Generalized Functions: Theory and Technique, Third Edition, Birkhäuser, Boston, 2004.

[11] Komatsu, H., Projective and injective limits of weakly compact sequences of locally convex spaces, J. Math. Soc. Japan 19 (1967), 366-383.

[12] Trèves, F., Topological Vector Spaces, Distributions, and Kernels, Academic Press, New York, 1967.

[13] van der Corput, J. G., Neutrices, J. Soc. Indust. Appl. Math., 7 (1959), 253279.

[14] Yang, Y. and Estrada, R., Regularization using different surfaces and the second order derivatives of 1/r, Appl. Anal. 92 (2013), 246-258.

[15] Yang, Y. and Estrada, R., Distributions in spaces with thick points, J. Math. Anal. Appl. 401 (2013), 821-835.

R. Estrada, Department of Mathematics, Louisiana State UniverSity, BATon Rouge, LA 70803, U.S.A.

E-mail address: restrada@math.1su.edu

J. Vindas, Department of Mathematics, Ghent University, Krijgslaan 281 Gebouw S22, B 9000 Gent, Belgium

E-mail address: jvindas@cage.UGent.be 